\title{
Learning about light and optics in on- line general education classes using at-home experimentation
}

Jacob Millspaw, Gang Wang, Mark Masters

Jacob Millspaw, Gang Wang, Mark F. Masters, "Learning about light and optics in on-line general education classes using at-home experimentation," Proc. SPIE 9289, 12th Education and Training in Optics and Photonics Conference, 92891M (17 July 2014); doi: 10.1117/12.2070527

SPIE Event: 12th Education and Training in Optics and Photonics Conference, 2013, Porto, Portugal 


\title{
Learning about light and optics in on-line general education classes using at-home experimentation.
}

\author{
Jacob Millspaw, Gang Wang, and Mark F. Masters \\ Department of Physics, Indiana University - Purdue University Fort Wayne (IPFW)
}

\section{INTRODUCTION}

College students are facing a constantly evolving educational system. Some still see mostly the traditional face to face lecture type classes where as others may never set foot on campus thanks to distance learning programs. In between they may enroll in a mix of face-to-face, two-way broadcasted interactive courses, streaming lecture courses, hybrid face-toface/on-line courses and the ominous MOOC! A large number of these non-traditional courses are general education courses and play an important role in developing non-science majors' understanding of science in general, and of physics in particular. We have been keeping pace with theses modern modes of instruction by offering several on-line courses such as Physics for Computer Graphics and Animation and Light and Color. These courses cover basic concepts in light, color and optics.

\section{GENERAL EDUCATION COURSES}

We strive to create an interactive ${ }^{1}$ learning environment in all of the physics courses in our program including the general education courses. It is important that the students realize that science (and learning in general) is an activity and not a passive experience. To encourage this we try to move away from traditional "experiments" (more like demonstrations) and have the students perform actual scientific investigations where they have to make sense of observational results or construct a related physical model. One can quickly see how distance and on-line learning might interfere with this instructional pedagogy.

Often we hear people raving about the usefulness of computers, tablets and other technological gadgets to improve the quality of our courses and to simply move them into a virtual world ${ }^{2}$. It has been our experience that for the most part this is not the case. These devices tend to be either a distraction or fail as a learning instrument and become just an overpriced content delivery system in an attractive box taking us back to a non-interactive sleepfest traditional course.

Another abomination occurring from the increase in on-line science courses is the death of the lab experience. There is some resistance to this by some who find clever ways for the students to perform meaningful experiments using household items and easy to use video analysis software such as Tracker ${ }^{3}$, but there are those who feel that virtual labs are the way to go especially when cost is considered. While these virtual labs may be chock full of conceptual ideas they are not labs; they are physical simulations.

We fall into the former camp if we have on-line courses then the students have REAL laboratories to do. We have designed inexpensive lab kits that allow the students to do a variety of table top at home (or in class) investigations related to light and optics. Combined with cell phone cameras and discussion boards we are able to keep our on-line general education classes hands on and interactive.

The topics covered in the Physics for Computer Graphics and Animation and Light and Color have a significant amount of overlap and include investigations related to additive/subtractive color mixing, the ray model of light, point and extended light sources, reflection, refraction, and image formation. Physics for Computer Graphics and Animation also utilizes the open source $3 \mathrm{~d}$ rendering software, Blender ${ }^{4}$, which allows the students to explore these topics in an idealized (with respect to light and color) virtual world. Their virtual observations can be compared to the physics investigations made with the kits providing insight into the problems faced when trying to create realistic rendered 
scenes in the CG world. The software also allows more control over surface coloration, reflectivity, roughness, and shape that is not available in the kits and is difficult to control experimentally. Light and Color covers the wave and particle nature of light.

\section{THE KIT AND ITS BITS AND PIECES}

The materials in the light exploration kits (Figure 1) include an LED controller, LEDs (including red, green, blue, white, UV and a 5 LED linear array), LED mounting stands, diffraction glasses, a mixing screen, filters (RGBCMY), absorption balls (RGBY), a set of apertures, foam board triangulation materials, cube and cylindrical refraction containers, a flexible mirror, 3 polarizers, glow paper and a peacock feather. The kit used for Physics for Computer Graphics and Animation does not include the items that are required for the investigations that are specific to only the Light and Color course.

\section{ADDITIVE AND SUBTRACTIVE COLOR MIXING}

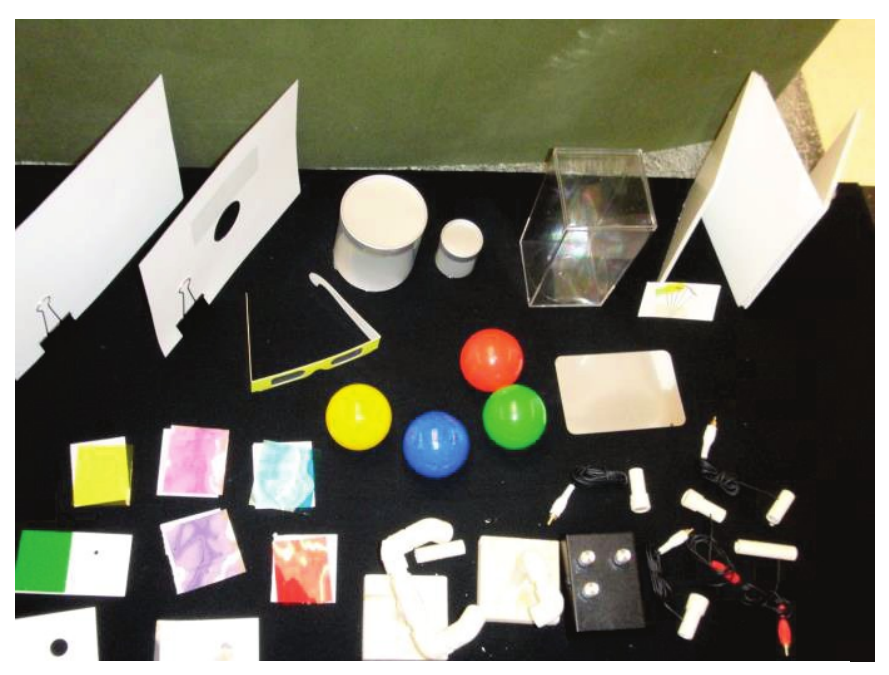

Figure 1: A layout of the components included in the Light and Color Exploration Kit.

The students are able to investigate color mixing in a variety of ways. They begin by exploring color through the diffraction glasses where they learn about color spectrum. Using the mixing screen (Figure 2a) students can compare the color spectrum of various light sources. Each LED can be compared to white LED for reference (Figure 2b) and through additive mixing of light from individual LEDs they can discover the difference between spectral and nonspectral colors. Figure 2c shows the results comparing the spectrum from an amber LED and the same color generated by additively mixing red and green light. All data is acquired by students using cell phone (or digital cameras)

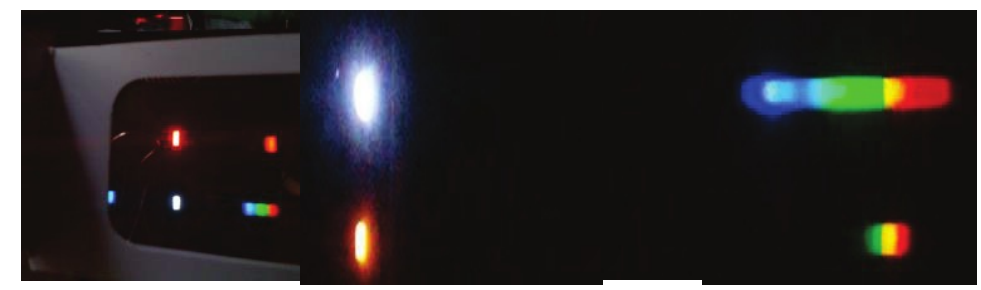

(a)

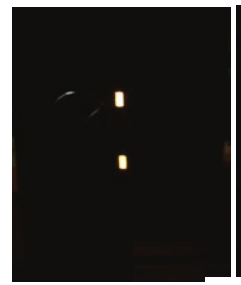

(b)

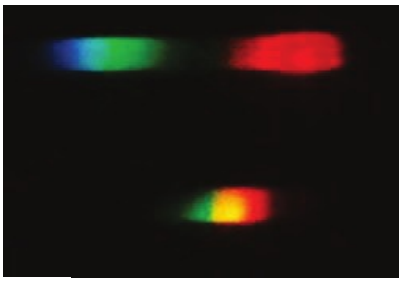

(c)

Figure 2: (a) A view of a red LED and a white LED through the diffraction glasses. (b) Students can compare their LEDs to the continuous spectrum produced by the white light. Notice that only part of the green and red spectrum is present in the amber LED. (c) The amber color can be produced from a mixture of red and green and it is clear that there is no yellow present in the mixed color spectrum seen on top.
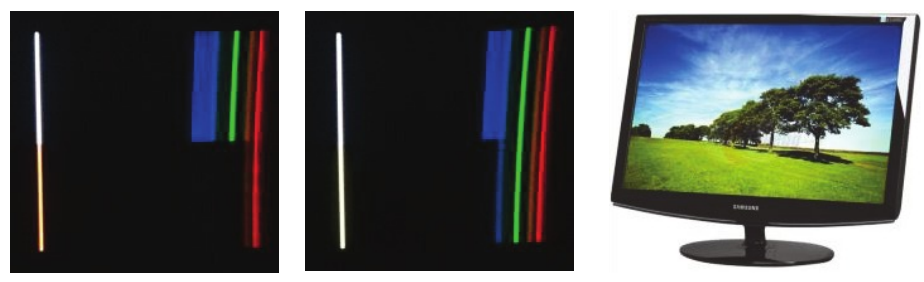

Figure 3: Various spectral lines can be observed through the diffraction glasses using an LCD monitor.
The students are encouraged to investigate various other light sources including their phones, TVs and light bulbs. In Figure 3 we see the spectrum produced from the backlight in an LCD monitor. The vertical line in the center is white on the top half for reference and the bottom half is changed to a series of colors. The observation of the white 
spectrum in this case show that the monitor is back lit with a CFL light. These observations help reinforce the importance of understanding light by demonstrating a common use of additive color mixing.

A traditional color mixing circle (Figure 4) can be produced using the apertures and variable brightness LEDs in the kits. Here students can explore what color combinations colors such as pink, lavender, orange and the elusive brown. We can

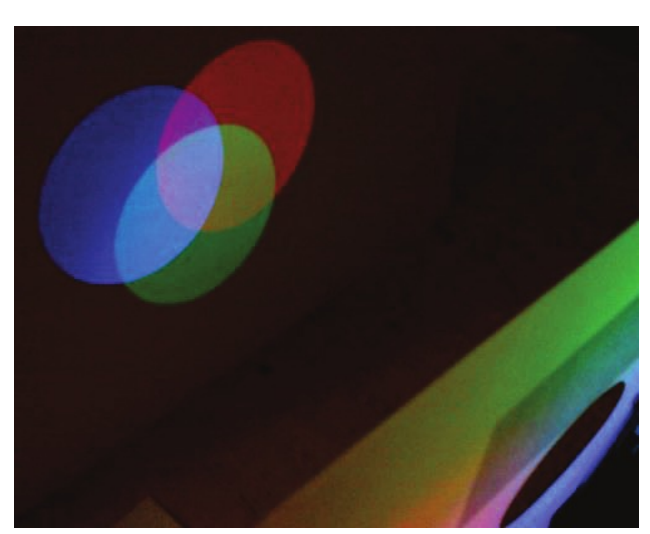

Figure 4: A traditional color mixing circle produced from RGB LEDs. also substitute a white light for one of the three colors and explore color saturation.

The kits allow for a variety of subtractive color mixing experiments as well. In Figure 5 you can see the results of introducing a variety of filter combinations. In Figure $\mathbf{6}$ we utilize the duck aperture with the RGB LEDs so students can qualitatively explore the effects of each filter on the individual colors. They quickly find that real filters do not behave in an ideal way! In this case the cyan filter does not completely remove the red light and it does remove a bit of the green light as well. The colored balls in the kit develop investigations related to subtractive color processes related to reflection of light. The use of various LEDs and filter combinations with objects around the home make for some interesting additional investigations.

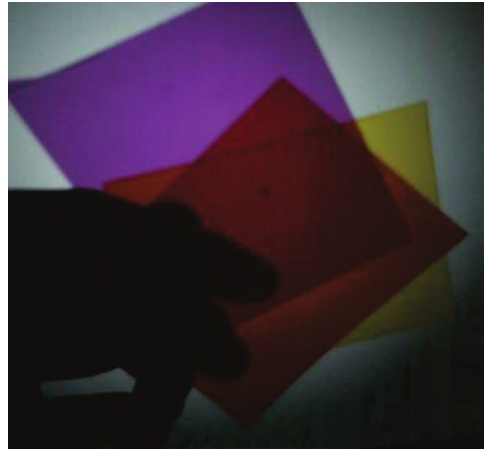

Figure 5: White light viewed through a combination of transmission filters.

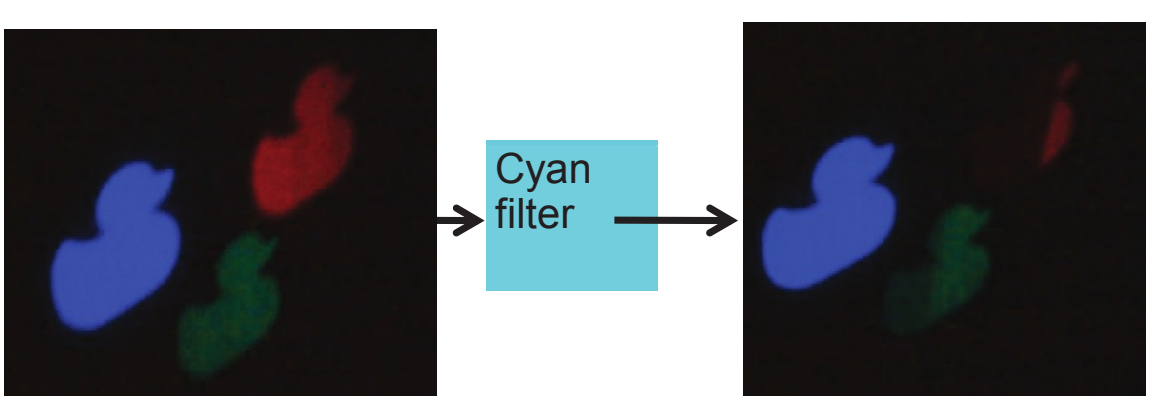

Figure 6: Configuring the RGB LEDs with the duck aperture allows students to see how each color is affected by the transmission filters. Note how red light is greatly diminished

From these investigations the students can develop an understanding of color mixing processes and use spectral data obtained from the diffraction glasses to predict results for various light/filter combinations. The students in Physics for Computer Graphics also produce simulations of each of the physical phenomena they observe. This involves their virtually manipulating different properties of solids and modeling different shapes.

Students can try their luck at solving the cubes (Figure 7). Here they must predict the colors of the cubes (Figure 7d) from images of the cubes illuminated with various colored sources (Figure 7a-c). 


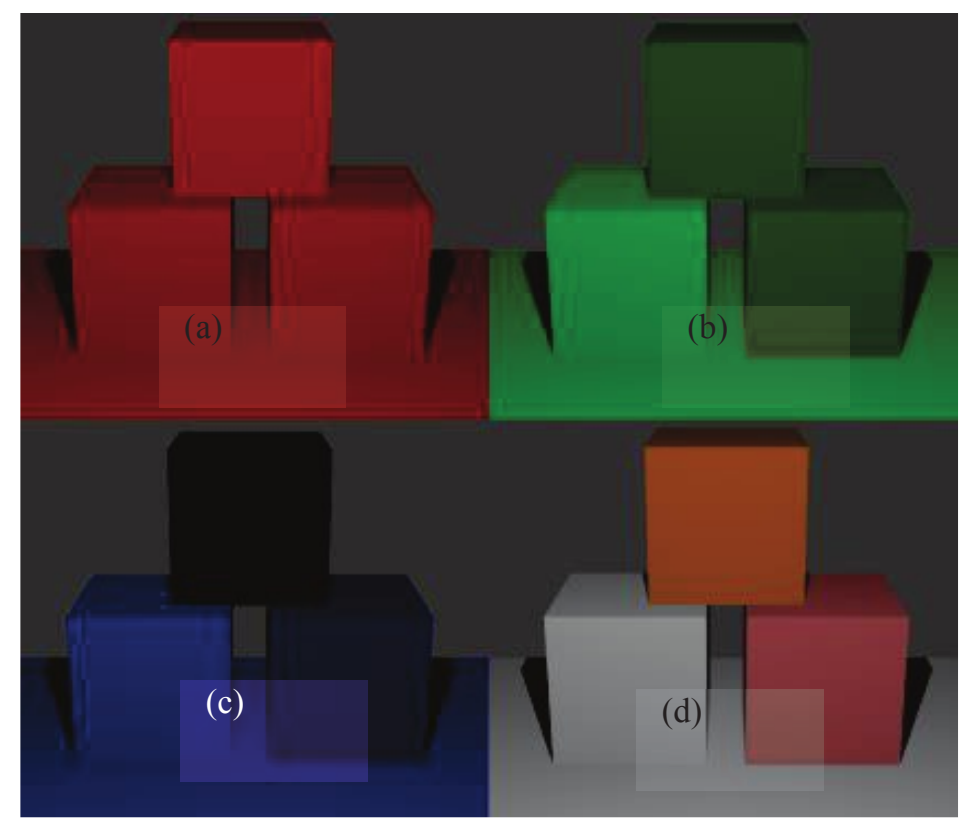

Figure 7: $(a, b, c)$ Color blocks viewed in simulated red, green and blue light. The students can attempt to predict the color of the blocks illuminated by white light (d) based on the observations and their understanding of the related subtractive process.

\section{RAY OPTICS: POINT AND EXTENDED SOURCES, REFLECTION AND REFRACTION}

Investigations into the ray nature of light can be done using the various apertures and light sources. The LEDs behave closely to point sources of light while the LED array acts as an extended source (Figure 8). After exploring with the circular apertures the students are asked to predict the results of illuminating a duck shaped aperture with both a point and extended source. Using materials from the kit or around the house they can cast shadows and learn about umbra and

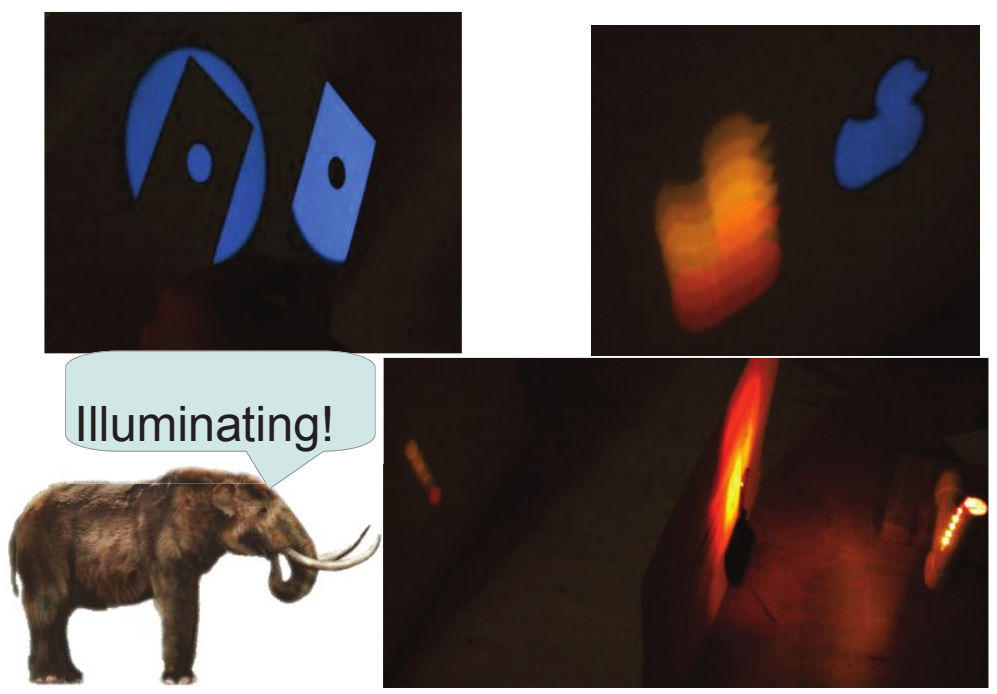

Figure 8: Investigations illuminating apertures with point and extended sources. penumbra (Figure 9).

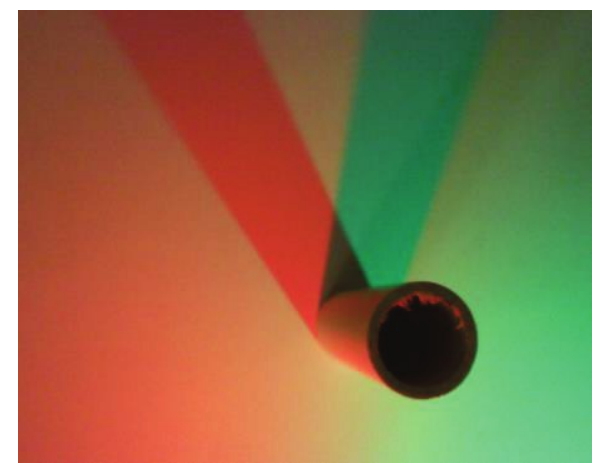

Figure 9: The umbra and penumbra can be easily observed using colored LEDs. 


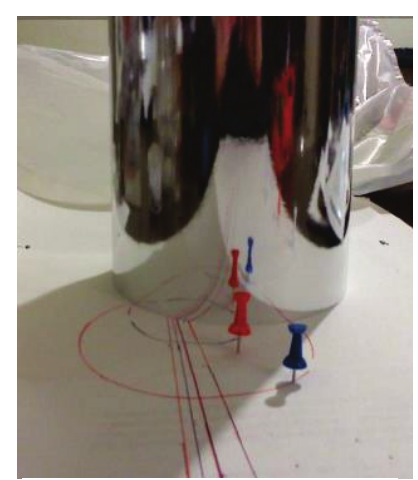

Figure 10: The flexible mirror.

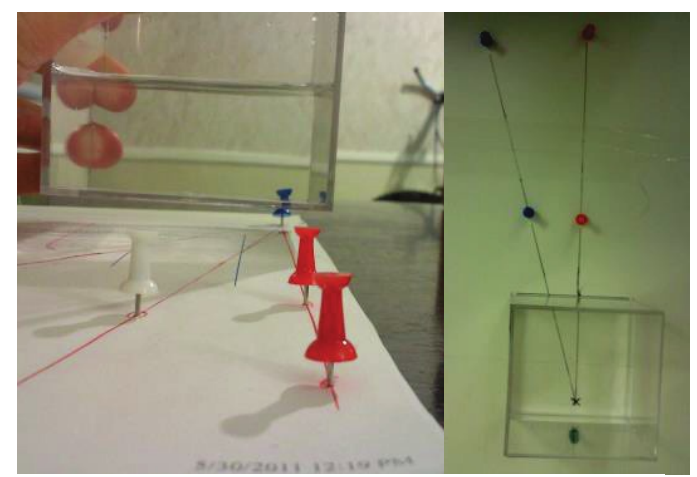

Figure 11: Ray tracing through the refraction cube.
The kit also contains a variety of equipment to perform ray tracing investigations related to reflection, refraction and image formation. The flexible mirror works as a flat surface mirror or a convex or concave cylindrical mirror (Figure 10). Both virtual and real images can be formed and located through triangulation. Students will also find a clear plastic cube and two different cylindrical containers that can be filled with water to explore refraction through flat or curved surfaces (Figure 11).

The students taking Physics for Computer Graphics and Animation must perform similar experiments within the virtual world produced through the $3 \mathrm{~d}$ rendering software. The image in Figure 12a is a rendered image of a pin triangulated through a refractive cube by lining up two virtual pins in front of the cube. In Figure 12b you see the top down location of the pins in the drawing environment. Notice the similarity to the experiment shown in Figure 11. The rendering software allows for analogs to every color mixing and ray tracing base investigation. This exposes the differences between the real world and the virtual environment. For example, in the virtual world it is quite easy to create ideal filters that pass $100 \%$ of a single color.

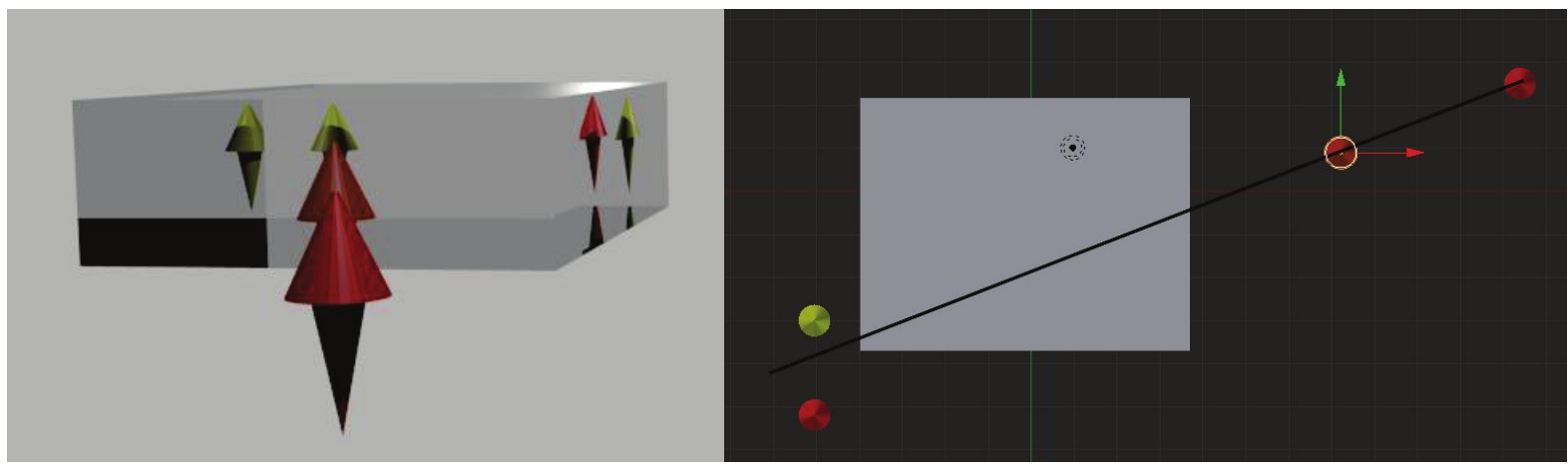

Figure 12: (a) A rendered view of triangulation through a refractive cube. (b) Top view showing the location of the virtual triangulation pins. Notice the similarity to the physical observations in Figure 11. There is also a bit of internal reflection in the rendered image.

\section{WAVE AND PARTICLE NATURES}

The Light and Color students explore the wave nature of light with a set of three linear polarizers (Figure 13). Students are encouraged to use the polarizers to make observations related to the polarization of light reflected off various surfaces such as bodies of water or just glare from the lights on a hall floor.

Interference is another fun wave-like behavior of light and what could be a more fun way to explore this than with iridescence from a peacock feather (Figure 14a). Iridescence is a commonly observed phenomenon connecting the physics to students' daily observations (Figure 14b). 


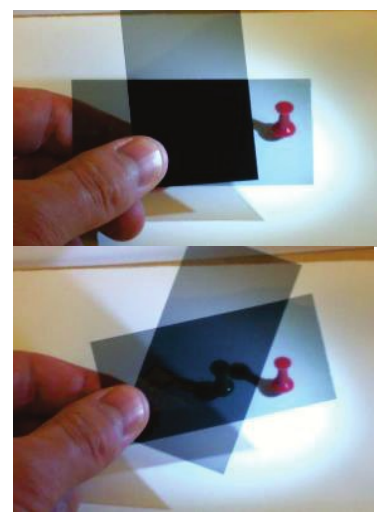

Figure 13: Linear Polarizers

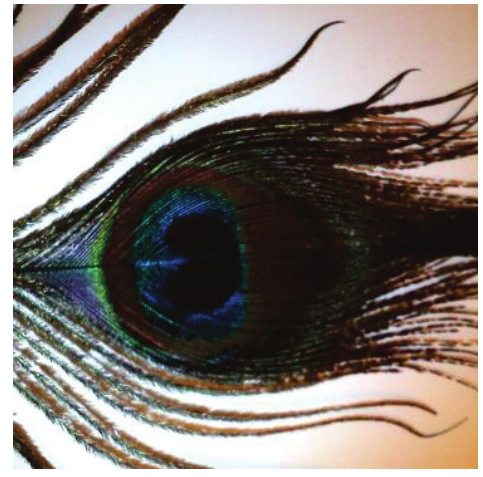

Figure 14: (a) Iridescence can be observed with the Peacock feather in various lighting. (b) Students can locate other iridescent objects outside of class.
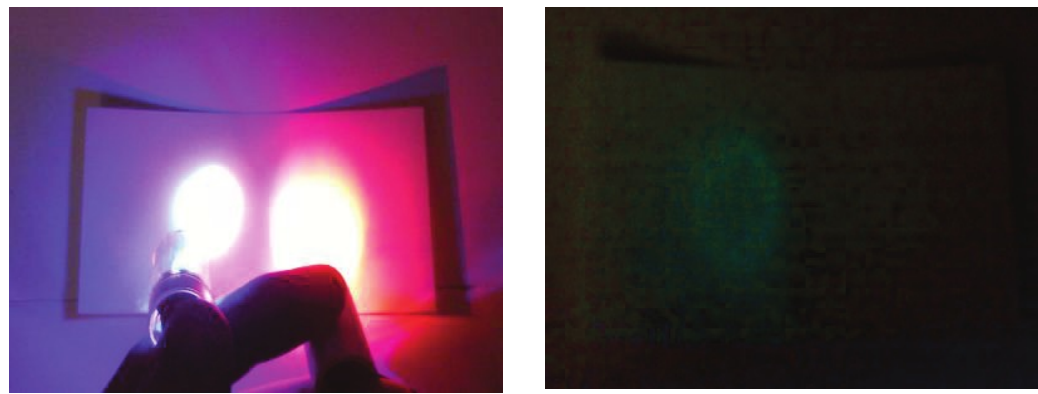

Lastly using a small piece of glow papers and the various LEDs the students can investigate a simple analog to the photo-electric effect. A piece of glow paper illuminated (Figure 15a) by a UV and a red LED will only glow under the area where the UV light was incident (Figure 15b) even if the intensity of the red LED is much higher than the UV.

Figure 15: (a) Glow paper illuminated with UV (left) and Red (right)

LEDs. (b) The paper glows green under the UV illuminated area.

\section{CONCLUSION}

The student response to the kits is positive. The general consensus is that working hands on with the concepts was more interesting and engaging than a traditional online course with video lectures. Many of the students reported that they were opting to keep the kits over selling them back so they could work through the experiments with their children. This is a great success.

No instructional method is without drawbacks. The assignments associated with the investigations were inquiry based and required students to record their observations and conclusions in short paragraphs. They were also required to include images of their experimental setups as well as their observations in their write-ups as well as posted into the discussion forums. This increased the amount of time required for grading.

We have also utilized the kits in 2-way video distances education sections as well as our face to face traditional Light and Color sections with positive results. The students seem more likely to answer questions related to observations they have made with their own eyes even if they are not $100 \%$ sure of the concepts than they are if queried on conceptual ideas when demonstrated by the instructor or simply discussed in class. The kits add a bit of a wow factor as many do not expect to see many of the results (e.g. red mixed with green producing yellow) making the class more energetic and engaged in learning.

1 Resource Letter ALIP--1: Active-Learning Instruction in Physics

David E. Meltzer and Ronald K. Thornton, Am. J. Phys. 80, 478 (2012), DOI:10.1119/1.3678299

2 A Year Without Paper: Tablet Computers in the Classroom

John Fons, Phys. Teach. 48, 481 (2010), DOI:10.1119/1.3488196

3 http://www.cabrillo.edu/ dbrown/tracker/

4 http://www.blender.org/ 